\title{
Optic Neuropathy
}

National Cancer Institute

\section{Source}

National Cancer Institute. Optic Neuropathy. NCI Thesaurus. Code C118711.

Disorder of the optic nerve. 